\title{
FROM A CONVERGENCE TO A REASONING WITH INTERNAL-VALUED PROBABILITY
}

\author{
PHAN DINH DIEU ${ }^{(1)}$ and TRAN DINH QUE ${ }^{(2)}$
}

\begin{abstract}
Combining a deduction in a knowledge base of external uncertainty whose semantics has been proposed by N. J. Nilsson with a deduction coming from a convergence of a sequence of operators in a knowledge base of internal uncertainty, we propose a method of reasoning in a knowledge base of the both types of uncertainty.

Let $B$ an $S$ be such a knowledge base and a goal sentence, respectively. The interval of truth probabilities of $S$ derived from $B$ can be found by the proposed method.
\end{abstract}

\section{INTRODUCTION}

This article presents a method of reasoning from a knowledge base with uncertain information represented in the form interval-valued probability.

Let $B$ be a knowledge base consisting of $B^{E}$ and $B^{I}$ in which $B^{E}$ is a knowledge base with external uncertainty whose element are given in the form $\langle S, I\rangle$, where $S$ is a sentence and $I=[a, b]$ is a closed subinterval of the unit interval $[0,1]$; and $B^{I}$ is a knowledge base with internal uncertainty whose elements are given the form

$$
\left\langle S_{1}, I_{1}\right\rangle \wedge \cdots \wedge\left\langle S_{n}, I_{n}\right\rangle \rightarrow\left\langle S_{n+1}, f\left(I_{1}, \ldots, I_{n}\right)\right\rangle
$$

where $S_{1}, \ldots, S_{n}, S_{n+1}$ are sentences; $I_{1}, \ldots, I_{n}$ are interval variables and $f$ : $C[0,1]^{n} \rightarrow C[0,1]$ is an interval function in which $C[0,1]$ is the set of all closed subintervals of the interval $[0,1]$.

Let $S$ be any given sentence. A semantics, with underlies a method of deducing the interval of truth probabilities of $S$ from $B$, will be given.

The article is structured as follows. In Section 2, we will consider a deduction from $B^{I}$ and particularly from a directed acyclic knowledge base (DAKB) to any sentence. Section 3 will briefly review a semantics of the probabilistic logic proposed by N. J. Nilsson, i.e., a method of reasoning in a knowledge base of extexnal uncertainty, and then devote mainly to a method of reasoning and conditions for deduction from a knowledge base containing both external and internal uncertainty.

\section{INTERNAL UNCERTAINTY}

Given a knowledge base $B=\left\{J_{j} \mid j=1, \ldots, M\right\}$, where $J_{j}$ is a rule of the form: 


$$
J_{j}=\left\langle A_{j_{1}}, I_{j_{1}}\right\rangle \wedge \cdots \wedge\left\langle A_{j_{m_{j}}}, I_{j_{m_{j}}}\right\rangle \rightarrow\left\langle A_{c_{j}}, f_{j}\left(I_{j_{1}}, \ldots, I_{j_{m_{j}}}\right)\right\rangle
$$

where $f_{j}: C[0,1]^{m_{j}} \rightarrow C[0,1]$ is an interval function from the Cartesian product $\underbrace{C[0,1] \times \cdots \times C[0,1]}_{m_{j} \text { times }}$ in to $C[0,1]$.

Let

$$
\Gamma\left(J_{j}\right)=\left\{A_{j_{1}}, \ldots, A_{j_{m_{j}}}, A_{c_{j}}\right\}
$$

and

$$
\Gamma=\bigcup_{J_{j} \in B} \Gamma\left(J_{j}\right)
$$

the set of all sentences in $B$. We define $I$ the set of all mappings from $\Gamma$ to $C[0,1]$. Such a mapping $I$ assigns to each sentence $P \in \Gamma$ an interval $I(P) \in C[0,1]$.

For the sake of simplicity, we denote $f_{j}(I)=f_{j}\left(I_{j_{1}}, \ldots, I_{j_{m_{j}}}\right),(j=1, \ldots, M)$, where $I \in I$ such that $I\left(A_{j_{i}}\right)=I_{j_{i}}, i=1, \ldots, m_{j}$.

An operator $t_{B}$ from $I$ to $I$ is defined as follows

$$
\begin{gathered}
t_{B}: I \rightarrow I \\
t_{B}(I)(P)=I(P) \cap \bigcap_{j \in E_{P}} f_{j}(I)
\end{gathered}
$$

for every $P \in \Gamma$, in which $E_{P}=\left\{j \mid A_{c_{j}}=P\right\}$ and we assume that $\bigcap_{j \in E_{P}} f_{j}(I)=$ $[0,1]$, whenever $E_{p}=\emptyset$. From the above, we can define recursively a sequence $\left\{t_{B}^{n}\right\}_{n \geq 0}$ as follows:

(i) $t_{B}^{0}(I)=I$;

(ii) $t_{B}^{n+1}(I)=t_{B}\left(t_{B}^{n}(I)\right)$ for every $I \in I$.

For any $I_{1}, I_{2} \in I$, we say that $I_{1} \leq I_{2}$ (respectively, $\left.I_{1}<I_{2}\right)$ iff $I_{1}(P) \subseteq$ $I_{2}(P)$ (respectively, $I_{1}(P) \subset I_{2}(P)$ ) for every $P \in \Gamma$. Accordingly, from the definition of the operator $t_{B}$, it is easy to see that $t_{B}^{n+1}(I) \leq t_{B}^{n}(I)$, for every $I \in I$ i.e., $\left\{t_{B}^{n}(I)(P)\right\}$, for any $I \in B$ and $P \in \Gamma$, is a sequence of closed subintervals satisfying the condition $\left.t_{B}^{n+1}(I)(P) \subseteq t_{B}^{n}(I)(P)\right\}$, for every $n$. Therefore, $\left\{t_{B}^{n}(I)\right\}$ is the convergent sequence under the meaning that $t_{B}^{\infty}(I)(P)=I^{*}(P)$, where $I^{*}(P)$ is a closed subinterval of the interval $[0,1]$ and it might be empty. Hence, we can define a convergence of the sequence $\left\{t_{B}^{n}\right\}$. However, it is not the case that for any $I \in I$ there always exits a number $n$ such that

$$
t_{B}^{n}(I)=t_{B}^{n+1}(I)
$$


For instance, let $B=\{A:[\alpha, \beta] \rightarrow A:[\sqrt{\alpha}, \sqrt{\beta}]\}$ and $I(A)=[a, 1],(0<a<1)$, then $t_{B}^{n}(I)(A)=\left[a^{\frac{1}{2 n}}, 1\right]$ It is clear that $t_{B}^{n} \neq t_{B}^{n+1}$ for every $n$.

Suppose now $B$ is a knowledge base as above. Let $\Gamma$ be the set of all sentences in $B$ and $D=\left\{D_{j_{r}}=\left(A_{j_{r}}, A_{c_{j}}\right) \mid r=1, \ldots, m_{j} ; j=1, \ldots, M\right\}$. Denote $\mathcal{G}=(\Gamma, D)$. We assume that there exist no cycles and loops, i.e., $(A, A) \notin D$ for every $A \in$ Igamma and no chain $\left(A_{i}, A_{i+1}\right) \in D, i=1, \ldots, r$ such that $A_{1}=A_{r+1}$. Remind that a graph is called a directed acyclic one iff it is directed and has no cycles and loops.

Therefore, $\mathcal{G}=(\Gamma, D)$ is composed of directed acyclic graphs $\mathcal{G}_{i}=\left(\Gamma_{i}, D_{i}\right)$, $i=1, \ldots, p$, where $\Gamma_{i}$ and $D_{i}$ are respectively sets of vertices and edges. We denote $\mathcal{G}=\mathcal{G}_{1} \cup \cdots \cup \mathcal{G}_{p}$ and also call $\mathcal{G}$ the graph of $B$ and $\Gamma=\bigcup_{i=1}^{p} \Gamma_{i}, D=\bigcup_{i=1}^{p} D_{i}$ the sets of vertices and edges, respectively; and every $\mathcal{G}_{i}$ is then called a component of the graph $\mathcal{G}$.

A knowledge base $B$ is called to be the directed acyclic knowledge base ( $D A K B$ ) iff the graph $\mathcal{G}$ of $B$ is a directed acyclic graph or is composed of directed acyclic graphs $\mathcal{G}_{i}, i=1, . ., p$.

In this article, we restrict our to the case that $B$ is a DAKB. Suppose that $B$ is such a knowledge base and $\mathcal{G}=(\Gamma, D)$ is its graph. Let $E_{A}=\{(B, A) \mid(B, A) \in$ $D\}$ and $E^{A}=\{(A, B) \mid(A, B) \in D\}$, then $\left|E_{A}\right|$ and $\left|E^{A}\right|$ are called the indegree and the outdegree of $A$, respectively (where $|\cdot|$ denotes cardinality). We denote $\operatorname{ind}(A)=\left|E_{A}\right|$ and $\operatorname{outd}(A)=\left|E^{A}\right|$.

Three types of vertices playing the important role afterwards will be named particularly:

(1) $A$ vertex $A$ is called the input vertex iff ind $(A)=0$;

(2) $A$ vertex $A$ is called the inside vertex iff ind $(A) \neq 0$ and $\operatorname{outd}(A) \neq 0$;

(3) $A$ vertex $A$ is called the output vertex iff outd $(A)=0$.

The following notion arises naturally from DAKB $B$ when the vertices of its graph are now combined with interval values.

A number $n$ is called the depth of a sentence $A$ in $\Gamma$ w.r.t. $I \in I$ if $n$ is the least number such that $t_{B}^{n}(I)(A)=t_{B}^{n+1}(I)(A)$. We denote $n=\operatorname{depth}_{B}(A, I)$.

It is clear that the computation of the interval value of a sentence $A$ from $B$ depends only on the component $\mathcal{G}_{i}$ containing it, especially on the type of vertex $A$ in $\mathcal{G}_{i}$. It is easy to prove the following.

Proposition 1. Let $B$ be a $D A K B$ and $I \in Z$. Then

(i) depth $\operatorname{di}_{B}(A, I)=0$ for every input vertex $A$;

(ii) $\operatorname{depth}_{B}(A, I) \geq \max _{B \in E_{A}}\left\{\operatorname{depth}_{B}(B, I)\right\}+1$, for every $A \in \Gamma$. 
Proposition 2. If $B$ is a $D A K B$, then there always exists a natural number $n$ such that $t_{B}^{n}(I)=t_{B}^{n+1}(I)$ for every $I \in I$.

Proof. Suppose that $\mathcal{G}=(\Gamma, D)$ is the graph of $B$, where $\mathcal{G}=\mathcal{G}_{1} \cup \cdots \cup \mathcal{G}_{p}$ is composed of components $\mathcal{G}_{i}=\left(\Gamma, D_{i}\right), i=1, \ldots, p$ and $\Gamma=\bigcup_{i=1}^{p} \Gamma_{i}, D=\bigcup_{i=1}^{p} D_{i}$. Let

$$
U_{i}^{k}=\left\{A \mid A \in \Gamma_{i} \text { and } \operatorname{depth}_{B}(A, I)=k\right\}
$$

where $k$ is the number of iterating times of $t$. It is clear that there will exist $n_{i},(i=1, \ldots, p)$ such that $U_{i}^{n_{i}}=\Gamma_{i}$. Taking $n=\max \left(n_{1}, \ldots, n_{p}\right)$, we have $t^{n}(I)=t^{n+1}(I)$. The proposition is proved.

From Proposition 2, we can define an operator $\tau_{B}$ as follows: For any $I \in I$, $\tau_{B}(I)=t_{B}^{n}(I)$, where $n$ is the least number such that $t_{B}^{n}(I)=t_{B}^{n+1}(I)$.

Suppose that $B$ is a directed acylic knowledge base composed of rules and $S$ is any sentence. We dentone by $\Gamma$ the set consisting of $S$ and all sentence occurring in rules $J_{i}$ of $B$. Let $I$ be a mapping which assigns a subinterval of the interval $[0,1]$ for any sentence in $\Gamma$. Then $\tau_{B}(I)(S)$ can be considered as the interval value for the truth probability of the sentence $S$ derived from the knowledge base $B$.

\section{REASONING WITH EXTERNAL AND INTERNAL UNCERTAINTY}

This section is devoted presenting a method of reasoning in a knowledge base with both forms of uncertaity: external and internal uncertaity. We first recall a semantics of reasoning in a knowledge base with external uncertainty, and then propose a decduction of knowledge base with containing both of uncertainty. Affer that we consider conditions under which the deduction may be obtained.

\subsection{A Method of Reasoning}

Given a knowledge base $B$ with external uncertainty

$$
B=\left\{\left\langle S_{i}, I_{i}\right\rangle \mid i=1, \ldots, L\right\}
$$

Let $\Gamma$ be the set of all sentences $S_{i}$ and $I=\{I \mid I: \Gamma \rightarrow C[0,1]\}$. We define an operator $R_{B}$ from $I$ to $I$ as follows.

For every $I \in I$, we establish a new knowledge base

$$
B^{\prime}=B \cup\{\langle P, I(P)\rangle \mid P \in \Gamma\}
$$

and we take for every $P \in \Gamma$ the interval $I^{\prime}(P)=\mathcal{F}\left(P, B^{\prime}\right)$, wich is deduced from $B^{\prime}$ - a deduction based on the sematics given by N. J. Nilsson (For more detail, 
refer to $[4,11])$. The mapping $I^{\prime}$ is defined to be the image of $I$ by the operator $R_{B}: R_{B}(I)=I^{\prime}$.

It is easy to see that

$$
R_{B}^{n}(I)=R_{B}(I), \text { for any } n \geq 1
$$

From now on, we consider knowledge bases containing both types of uncertainty: external and internal uncertainty. Let $B$ be such a knowledge base, we can write $B=B^{E} \cup B^{I}$, where $B^{E}$ consists of knowledge with external uncertainty, and $B^{I}$ contains knowledge with internal uncertainty.

Suppose that

$$
\begin{aligned}
B^{E} & =\left\{\left\langle S_{i}, I_{i}\right\rangle \mid i=1, \ldots, L\right\} \\
B^{I} & =\left\{J_{j} \mid j=1, \ldots, M\right\}
\end{aligned}
$$

where

$$
J_{j}=\left\langle A_{j_{1}}, I_{j_{1}}\right\rangle \wedge \cdots \wedge\left\langle A_{j_{m_{j}}}, I_{j_{m_{j}}}\right\rangle \rightarrow\left\langle A_{c_{j}}, f_{j}\left(I_{j_{1}}, \ldots, I_{j_{m_{j}}}\right)\right\rangle
$$

and $S$ is any (target) sentence. Our problem is to compute the interval value for the truth probability of the sentence $S$ from the knowledge base $B$.

We put $\Gamma$ to be the set of all mapping from $\Gamma$ to $C[0,1]$.

Let $I_{0}$ be the mapping defined by

$$
I_{0}= \begin{cases}I_{i} & \text { if } P=S_{i} \text { for some } i=1, \ldots, L \\ {[0,1]} & \text { otherwise }\end{cases}
$$

$I_{0}$ is called the initial assignment (of interval values to sentences in $\Gamma$ ).

We now define a sequence of assignments $I_{n}(n=0,1 \ldots)$ initiated by $I_{0}$ and given recursively as follows

$$
I_{n}= \begin{cases}R\left(I_{n-1}\right) & \text { if } n \text { is odd } \\ \tau\left(I_{n-1}\right) & \text { if } n \text { is positive even }\end{cases}
$$

Here $R$ and $\tau$ stand for $R_{B E}$ and $\tau_{B E}$, respectively.

Let $n$ be the least number having the property $I_{n}=I_{n+1}=I_{n+2}$ (if there exists). We denote this $I_{n}$ by $I^{*}$ and call it to be the resulting assignment deduced from $B$ to sentences in $\Gamma$. The interval $I^{*}(S)$ is defined to be the interval value for the truth probability of the sentence $S$ derived from the knowledge $B$. We also write:

$$
B \vdash\left\langle S, I^{*}(S)\right\rangle
$$

We will clarify the sematics of deduction by the following example. 
Example 1. Suppose that $B=B^{E} \cup B^{I}$, where $B^{E}$ is the set of sentences

$$
\begin{aligned}
B \rightarrow A & :[1,1] \\
A \rightarrow C & :[1,1] \\
B & :[.2, .8] \\
C: & {[.4, .7] }
\end{aligned}
$$

and $B^{I}$ is the set of rules

$$
\begin{aligned}
& J_{1}=C:\left[x_{1}, y_{1}\right] \rightarrow B:\left[\sqrt{x_{1}}, \sqrt{y_{1}}\right] \\
& J_{2}=B:\left[x_{2}, y_{2}\right] \wedge C:\left[x_{3}, y_{3}\right] \rightarrow A:\left[x_{2}, y_{2}\right]
\end{aligned}
$$

Calculate the interval of truth probabilities of the sentences $A$.

Step 1. Applying the operator $R$, we get

$$
\begin{aligned}
& A:[.2, .7] \\
& B:[.2, .7] \\
& C:[.4, .7]
\end{aligned}
$$

Step 2. The operator $\tau$ is applied

$$
\begin{aligned}
& A:[\sqrt{.4}, \sqrt{.7}] \\
& B:[\sqrt{.2}, \sqrt{.7}] \\
& C:[.4, .7]
\end{aligned}
$$

It is easy to see that after iterating $R$, then $\tau$, the interval values of $A, B, C$ are not changed. So we get the result $A:[\sqrt{.4}, \sqrt{.7}]$.

\subsection{Conditions of Deduction}

In general, it is not the case that there always exists a number $n$ such that $I^{*}=I_{n}=I_{n+1}=I_{n+2}$. In effect, we consider the following example.

Example 2. Suppose

$$
\begin{aligned}
B^{E} & =\{P \rightarrow Q:[1,1], P:[a, 1]\} \quad(0<a<1), \\
B^{I} & =\{Q:[x, y] \rightarrow P:[\sqrt{x}, y]\} .
\end{aligned}
$$


by simply computing, we have

$$
\begin{aligned}
& I_{0}(P)=I_{1}(P)=[a, 1] \\
& I_{n}(P)=\left[a^{\frac{1}{2^{n-1}}}, 1\right], n \geq 1
\end{aligned}
$$

and therefore $I_{n} \neq I_{n+1}$ for every $n \leq 1$.

By replacing the part $B^{E}$ of the knowledge base $B$ with

$$
B^{E}=\{P \rightarrow Q:[1,1], P:[a, b]\} \quad(0<a<b<1)
$$

and then $I_{n}(P)=\left[a^{1 \text { over } 2^{n-1}}, b\right], n \geq 1$. Thus, there exists a number $n$ such that $a^{\frac{1}{2^{n-1}}}>b$ or $I_{n}(P)=\emptyset$, for some $n$, i.e., $B=B^{E} \cup B^{I}$ is inconsistent.

Our problem is now to look for conditions guatanteeing that there exists a number $n$ such that $I_{n}=I_{n+1}=I_{n+2}$. The folowing proposition hold obviously.

Proposition 3. If $B=B^{E} \cup B^{I}$ is a knowledge base in which $S_{i} \neq A_{c_{j}}$ for nay $i=1, \ldots, L$ and $j=1, \ldots, M$, then there always exists the resulting assignment $I^{*}$ from $B$.

We call an interval function $f_{i}$ to be non-increasing (respectively, increasing) if for every $I_{1}, I_{2} \in I, I_{1}<I_{2}$ then $f_{j}\left(I_{1}\right) \supseteq f_{j}\left(I_{2}\right)$ (respectively, $F_{j}\left(I_{1}\right) \subset f_{j}\left(I_{2}\right)$.

Proposition 4. If $f_{j}(j=1, \ldots, M)$ is a non-increasing function, there always exits the resulting assignment $I^{*}$ from $B$.

Proof. We can write

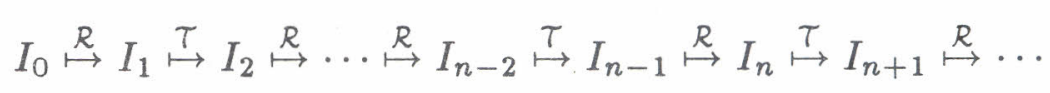

From the definition of the operator $t$, we have

$$
I_{1}^{\prime}(P)=t\left(I_{1}\right)(P)=I_{1}(P) \cap \bigcap_{j \in E_{F}} f_{j}\left(I_{1}\right)
$$

for every $P \in \Gamma$. In the case that $I_{1}^{\prime}(P)=I_{1}(P)$, for any $P \in \Gamma$, then $I^{*}=I_{1}$, as desired; otherwise, again applying the operator $t$ to $I_{1}^{\prime}$ we get

$$
\begin{aligned}
t^{2}\left(I_{1}\right)(P) & =t\left(I_{1}^{\prime}\right)(P) \\
& =I_{1}^{\prime}(P) \cup \bigcap_{j \in E_{P}} f_{j}\left(I_{1}^{\prime}\right) \\
& =I_{1}(P) \cap \bigcap_{j \in E_{P}} f_{j}\left(I_{1}\right) \cap \bigcap_{j \in E_{P}} f_{j}\left(I_{1}^{\prime}\right)
\end{aligned}
$$


By virtue of that $I_{1}>I_{1}^{\prime}$, we have $f_{j}\left(I_{1}\right) \subseteq f_{j}\left(I_{1}^{\prime}\right)$ Consequently,

$$
t^{2}\left(I_{1}\right)(P)=I_{1}(P)=I_{1}(P) \cap \bigcap_{j \in E_{P}} f_{j}\left(I_{1}\right)=t\left(I_{1}\right)(P)
$$

for every $P \in \Gamma$. Therefore, $I_{2}=\tau\left(I_{1}\right)=t\left(I_{1}\right), R\left(I_{2}\right)=I_{3}$. It is clear that from the inclusions

$$
I_{3}(P) \subseteq t\left(I_{1}\right)(P) \subseteq \bigcap_{j \in E_{P}} f_{j}\left(I_{1}\right) \subseteq \bigcap_{j \in E_{P}} f_{j}\left(I_{3}\right)
$$

follows

$$
\begin{aligned}
I_{3}^{\prime}(P) & =t\left(I_{3}\right)(P) \\
& =I_{3}(P) \cup \bigcap_{j \in E_{P}} f_{j}\left(I_{3}\right) \\
& =I_{3}(P), \text { for every } P \in \Gamma
\end{aligned}
$$

Hence, $\tau\left(I_{3}\right)=I_{3}$ and it is obvious that $R\left(I_{3}\right)$. So, $I^{*}=I_{3}$, as desred. The proof is complete.

Turing to Example 1 in Section 3.1, we see that although the knowledge base $B$ does not satisfy conditions of proposition 3-4, there exists the resulting assignment $I^{*}$ from $B$. So, it seems that the existence of $I^{*}$ depends strongly not only on properties of classes of functions $\left\{f_{j}\right\}$, but also on "syntax structure" of sentences in $\Gamma$. The problem of finding sufficient and necessary conditions for the existence of decduction and that of handling inconsistency are the subjects of our further work.

\section{REFERNCES}

1. J.F. Baldwin, Evidential Support Logic Programming. J. Fuzzy Sets and Systems, 24 (1987) 1-26.

2. P.D. Dieu, Probabilistic Logic Appoximate Reasoning. In I. Palander, editors, Artificia Interlligence and Information Control Systems of Robots-89, papes 107-112, North Holland, 1989.

3. P.D. Dieu, On a Theory of Interval-value Probabilistic Logic. Reseach Report, NCSR Vietnam, Hanoi, 1991.

4. P.D. Dieu and T.D. Que, Reasoning in Knowledge Base with External and Internal Uncertaity. J. Computer Science and Cybernetics, 2 (1994) 1-8.

5. P. D. Dieu and P. H. Giang, Interval-valued Probabilistic Logic for Logic Programs. J. Computer Science and Cybernetics, 3 (1994) 1-12.

6. R. Fagin, J. Y. Halpern, and N. Megiddo, A Logic for Reasoning about Probabilitis. J. Information and Computation, 87 (1990) 78-128. 
7. A. M. Frish and P. Haddaway, Anytime Deduction for Probabilitis Logic. October 21, 1992 (To appear in Artifical Intellgence).

8. L. van der Gaag, Computing Probability Intervals under Independency Constraints. In P. P. Bonissone, M. Henrion, L. N. Kanal, and J.F. Lemmer, editors, Uncertainty in Artificial intelligence 6, pages 457-466, Elsevier Science Publ., 1991.

9. M. R. Genesereth and N. J. Nilsson, Logical Foundations of Artifical Intelligence. Morgan Kaufmann Publ., Los Altos, CA, 1987.

10. R. Kruse, E. Schwecke, and J. Heisohn, Uncertainty and Vaguenss in Knowledge Based Systems. Numerical Methods. Spinger Varlag Publ., 1991.

11. N. J. Nilsson, Probalibistic Logic. J. Artificial Intellgence, 28 (1986) 71-87.

12. Ng. Raymond and V.S. Subramanian, Probabilistic Logic Programming. J. Information and computation, 101 (1992) 150-201.

(1) Institute of Information Technology

Nghia Do, Cau Giay, Hanoi, Vietnam.

(2) Department of Mathematics and Computer Science

Hue University, Hue, Vietnam.

Received: January 15, 1997 